\title{
CXCL13, CCL4, and sTNFR as circulating inflammatory cytokine markers in primary and SLE-related autoimmune hemolytic anemia
}

\author{
Boting $\mathrm{Wu}^{1,2}$, Weiguang Wang ${ }^{1}$, Yanxia Zhan ${ }^{1}$, Feng $\mathrm{Li}^{1}$, Shanhua Zou' ${ }^{1}$, Lihua Sun ${ }^{4^{*}}$ and Yunfeng Cheng ${ }^{1,3,4^{*}}$
}

\begin{abstract}
Background: A considerable proportion of autoimmune hemolytic anemia (AlHA) are secondary to underlying autoimmune disorders, especially syetemic lupus erythematosus (SLE), and the clinical and laboratory index for early discrimination between primary and SLE-related AlHA has yet to be defined. In the present study, we proposed novel cytokine patterns in the pathogenesis of AlHA as well as parameters for the timely identification of SLE-related patients.
\end{abstract}

Methods: AlHA patients confirmed by immunohematology techniques from September 2010 to December 2012 in our facility were consecutively included and categorized into primary $(n=19)$ and SLE-related $(n=18)$ groups. Plasma cytokine profiles were measured in a single procedure by Quantibody Human Inflammatory Array 1 (RayBiotech, Norcross, GA).

Results: SLE-related AlHA patients demonstrated younger age ( $39 \pm 20$ vs.57 \pm 16 years, $p=0.004$ ), poorer reticulocyte compensation ( $6.8 \pm 7.1$ vs.12.2 $\pm 8.6 \%, p=0.045)$, lower levels of lactate dehydrogenase [361 (265-498) vs. 622 (387-1154) U/L, $p=0.004]$, and higher occurrence of anticardiolipin antibody [9/18 (50\%) vs. 2/19 (10.9\%), $p=0.009]$. MCP-1/CCL2, MIP-1 $\beta / C C L 4, B L C / C X C L 13, ~ I L-8 / C X C L 8$, sTNFRI, and sTNFRII were significantly up-regulated in both groups, while sTNFRII was remarkably higher in SLE-related patients. Among both groups, hemoglobin level was negatively correlated with $\mathrm{CXCL} 13(r=-0.332, \mathrm{p}=0.044)$, while reticulocyte count was positively correlated with CCL4 ( $r=0.456, p=0.005)$.

Conclusion: CXCL13 and CCL4 could act as circulating biomarkers in AlHA, and indicated disease severity and erythroid compensation, respectively. Higher plasma sTNFRII might favor the diagnosis of SLE-related instead of primary AlHA.

Keywords: Autoimmune hemolytic anemia, Systemic lupus erythematosus, CXCL13, CCL4

\section{Background}

Autoimmune hemolytic anemia (AIHA), classified into warm AIHA and cold agglutinin syndrome, are characterized by robust erythrocyte autoantibody production to various extents. As high as $50 \%$ of warm AIHA and $90 \%$ of cold agglutinin syndrome are considered to be secondary to infections, malignancies, and systemic autoimmune disorders including systemic lupus erythematosus (SLE), thus hindering the understanding of key immune aberrations of

\footnotetext{
* Correspondence: qpsunlh023@126.com; yfcheng@fudan.edu.cn

${ }^{4}$ Department of Hematology, Zhongshan Hospital Qingpu Branch, Fudan

Universiy, Shanghai 201700, China

'Department of Hematology, Zhongshan Hospital Fudan University,

Shanghai 200032, China

Full list of author information is available at the end of the article
}

AIHA [1-3]. The autoantibody-centered concept for the pathogenesis of AIHA has been complemented, if not entirely altered by accumulating understandings of $\mathrm{T}$ helper cell and its functional subgroups. Previous studies revealed elevation of IL-10 production in both peripheral blood and spleen Th population, and suggested potential role of Treg and Th1 cells in AIHA [4-6]. However, the exact dominating Th functional population is yet to be identified in AIHA, which allows us to look for additional circulating cytokine markers that might have played potential roles in the pathogenesis of AIHA. The present study utilized a multiplex detection system to illustrate plasma cytokine portraits in primary and SLE-related AIHA patients, thus 
intending to identify novel inflammatory cytokine markers in this highly heterogeneous disease group.

\section{Materials and methods Study population}

AIHA patients confirmed by immunohematology techniques including direct antiglobulin test and gel microcolumn antiglobulin assay from September 2010 to December 2012 in our facility were consecutively included. Patient history and baseline laboratory parameters were collected by a hematologist at the time of diagnosis. Additional panel of autoantibodies including anti-nuclear antibody, anticardiolipin antibody, and extractable nuclear antibodies were performed in all patients, and the diagnosis of SLE was made according to the American College of Rheumatology criteria [7]. When overt lymphadenopathy was present and lymphoid malignancies suspected, lymphadenectomy biopsy was performed to confirm or exclude the diagnosis. The health control group comprised of 10 healthy adults (6 males and 4 females) at median age of 34 (24-46) years without known history of malignancies, autoimmune diseases, or recent infections. The study was approved by Medical Ethics Committee of Zhongshan Hospital of Fudan University. Written informed consent was obtained from each patient before being included in the study.

\section{Plasma inflammation cytokine array}

Cytokine profiles were measured by Quantibody Human Inflammatory Array 1 (RayBiotech, Norcross, GA) which permitted detection of 40 inflammation-associated cytokines including IFN- $\gamma$, I-309/CCL1, MCP-1/CCL2, MIP$1 \alpha / C C L 3, \quad M I P-1 \beta / C C L 4$, MIP-1 $\delta / C C L 15$, RANTES/ CCL5, Eotaxin/CCL11, Eotaxin-2/CCL24, MIG/CXCL9, BLC/CXCL13, G-CSF, M-CSF, GM-CSF, ICAM-1, IL-1 $\alpha$, IL-1 $\beta$, IL-1ra, IL-2, IL-4, IL-5, IL-6, IL-6sR, IL-7, IL-8/ CXCL8, IL-10, IL-11, IL-12p40, IL-12p70, IL-13, IL-15, IL-16, IL-17, TIMP-1, TIMP-2, PDGF-BB, TNF $\alpha$, TNF $\beta$, sTNFRI and sTNFRII in a single procedure using plasma samples cryopreserved at $-80^{\circ} \mathrm{C}$. The protein array slides spotted by specific capture antibodies were incubated with thawed plasma samples, washed, and incubated with a cocktail of biotinylated antibodies using protocol provided by the manufacturer. The slides with bound biotin were then incubated with streptavidin-conjugated Hylite Plus 555 fluor. Relative fluorescent strength was detected by LuxScan 10 K-A Microarray Scanner (CapitalBio Corporation, Beijing, China). The actual protein concentration was obtained on the standard curve plotted via standard controls incorporated into the array.

\section{Statistical analyses}

Analysis was performed with the SPSS 11.5 software (SPSS, Chicago, IL, USA). Data were reported as mean \pm standard deviation or medians (inter-quartile ranges) for continuous variables and as frequencies (percentages) for categorical variables. Continuous variables between groups were assessed by the one-way ANOVA or MannWhitney U-test as appropriate. Differences in percentages were evaluated using the $\chi^{2}$ tests or Fisher's exact tests. The correlation between circulating cytokine levels and clinical parameters was calculated by Pearson's correlation coefficient or Spearman rank correlation coefficient as appropriate. Statistical significance was defined as two-sided $\mathrm{P}<0.05$. The level of significance for pairwise comparisons was adjusted when multiple comparisons were performed $(\mathrm{p}=0.05 / 3=0.017)$.

\section{Results and discussion}

Clinical characteristics of primary and SLE-related AIHA

From September 2010 to December 2012, 46 patients were serologically confirmed as warm antibody AIHA in our facility, among which 18 patients were later categorized as SLE-related AIHA, 7 lymphoma-related AIHA (4 angioimmunoblastic $\mathrm{T}$ cell lymphomas, 2 chronic lymphocytic leukemias, and 1 nodal marginal zone lymphoma), 1 drug-induced AIHA, 1 related to nonhematological malignancy, and the rest 19 patients were categorized as primary AIHA.

SLE-related AIHA patients were significantly younger than primary cases at the time of diagnosis ( $39 \pm 20$ vs.57 \pm 16 years, $p=0.004$ ), meanwhile the serological parameters for hemolysis including lactate dehydrogenase [361 (265-498) vs. 622 (387-1154) U/L, p = 0.004], total bilirubin $(1.4 \pm 1.4$ vs. $3.1 \pm 1.7 \mathrm{mg} / \mathrm{dl}, \mathrm{p}=0.002)$, and conjugated bilirubin $(0.7 \pm 0.7 \mathrm{vs} .1 .9 \pm 1.3 \mathrm{mg} / \mathrm{dl}, \mathrm{p}=0.002)$ were less prominently elevated. Gender distribution, severity of anemia, and platelet count were similar between SLErelated and primary AIHA patients, although reticulocyte count seemed to be lower in SLE-related cases (6.8 \pm 7.1 vs. $12.2 \pm 8.6 \%, \mathrm{p}=0.045)$. Except for anticardiolipin antibody [9/18 (50\%) vs. $2 / 19(10.9 \%), \mathrm{p}=0.009]$, major immunological parameters including complement levels, anti-nuclear antibody, direct antiglobulin test, indirect antiglobulin test, and peripheral lymphocyte phenotype were all comparable between SLE-related and primary AIHA patients (Table 1).

\section{Plasma cytokine portraits of primary and SLE-related AIHA}

Within the detection panel of inflammation-associated cytokines measured, MCP-1/CCL2, MIP-1ß/CCL4, IL-8/ CXCL8, BLC/CXCL13, sTNFRI and sTNFRII were found to be significantly up-regulated in both SLE-related and primary AIHA patients, while sTNFRII was remarkably higher in SLE-related cases. On the other hand, established cytokine markers for renowned Th functional subgroups such as Th1 (IFN- $\gamma)$, Th2 (IL-4), and Th17 (IL-17) were stable, 
Table 1 Baseline characteristics of primary and SLE-related AIHA patients

\begin{tabular}{|c|c|c|c|}
\hline & Primary $(n=19)$ & SLE-related $(n=18)$ & $\mathrm{p}$ value \\
\hline Age, years & $57 \pm 16$ & $39 \pm 20$ & $0.004 \%$ \\
\hline Female gender & $9(47.4)$ & $13(72.2)$ & 0.124 \\
\hline Hemoglobin, g/dl & $6.9 \pm 1.9$ & $7.9 \pm 2.6$ & 0.199 \\
\hline Reticulocyte count,\% & $12.2 \pm 8.6$ & $6.8 \pm 7.1$ & $0.045^{\S}$ \\
\hline Platelet count, $\times 10^{9} / \mathrm{L}$ & $166 \pm 104$ & $171 \pm 101$ & 0.874 \\
\hline Total bilirubin, mg/dl & $3.1 \pm 1.7$ & $1.4 \pm 1.4$ & $0.002 \%$ \\
\hline Unconjugated bilirubin, mg/dl & $1.9 \pm 1.3$ & $0.7 \pm 0.7$ & $0.002 \%$ \\
\hline Lactate dehydrogenase, U/L & $622(387-1154)$ & $361(265-498)$ & $0.004 \%$ \\
\hline \multicolumn{4}{|l|}{ Complements } \\
\hline $\mathrm{C} 3, \mathrm{mg} / \mathrm{dl}$ & $73.6 \pm 31.8$ & $56.5 \pm 36.7$ & 0.296 \\
\hline $\mathrm{C} 4, \mathrm{mg} / \mathrm{dl}$ & $9.4 \pm 5.5$ & $7.2 \pm 6.7$ & 0.451 \\
\hline \multicolumn{4}{|l|}{ Direct antiglobulin test } \\
\hline Anti-lgG (+) & $19(100)$ & $18(100)$ & 1.000 \\
\hline Anti-C3d (+) & $15(78.9)$ & $17(94.4)$ & 0.370 \\
\hline \multicolumn{4}{|l|}{ Autoantibodies } \\
\hline Antinuclear antibody (+) & $13(68.4)$ & $17(94.4)$ & 0.110 \\
\hline Anticardiolipin antibody (+) & $2(10.5)$ & $9(50.0)$ & $0.009 *$ \\
\hline Indirect antiglobulin test, titer & $8(1-32)$ & $2(1-4)$ & 0.169 \\
\hline \multicolumn{4}{|l|}{ Peripheral lymphocyte phenotype } \\
\hline $\mathrm{CD}^{+} \mathrm{CD}^{+}, \%$ & $36 \pm 20$ & $34 \pm 9$ & 0.804 \\
\hline $\mathrm{CD}^{+} \mathrm{CD}^{+}, \%$ & $33 \pm 18$ & $40 \pm 11$ & 0.294 \\
\hline $\mathrm{CD} 16^{+} \mathrm{CD} 56^{+}, \%$ & $7 \pm 5$ & $7 \pm 4$ & 0.984 \\
\hline $\mathrm{CD} 19^{+}, \%$ & $21 \pm 12$ & $17 \pm 7$ & 0.365 \\
\hline
\end{tabular}

Data are presented as mean $\pm \mathrm{SD}$, as number (percentage), or as median (interquartile range).

$\S_{p}<0.05, \aleph_{p}<0.01$.

or even markedly down-regulated, in both AIHA patient groups. (Table 2, Figure 1)

\section{Correlation between plasma cytokines and clinical manifestation in AlHA}

Correlation analyses between plasma cytokine levels and clinical parameters including hemoglobin level, reticulocyte count, and lactate dehydrogenase were performed among 37 AIHA patients of both primary and SLErelated genre (Figure 2). Among the significantly upregulated cytokines, CXCL13 was found to be negatively correlated with hemoglobin level $(\mathrm{r}=-0.332, \mathrm{p}=0.044)$, while positive correlation was found between CCL4 and reticulocyte count $(\mathrm{r}=0.456, \mathrm{p}=0.005)$.

\section{Discussion}

As one of the earliest acknowledged autoimmune disorders, warm antibody AIHA occurs in 1.25-2.6:100,000 persons per year [1,2]. The diagnostic panel of AIHA is mainly based upon immunohematological methods developed since mid $20^{\text {th }}$ century. Our understanding of the pathogenesis of AIHA is still limited except for its high prevalence of autoantibody. Fagiolo et al. reported enhanced IL-10 but decreased IL-12 production among peripheral blood mononuclear cells from primary AIHA patients, which indicated IL-12/IL-10 imbalance and possible Th1/Th2 dysregulation [4]. However, the repeatedly observed IL-10 upregulation was later attributed to regulatory $\mathrm{T}$ cells for Th1 inhibition, and the key Th functional subgroup was argued to be Th1 instead of Th2 or Th17 $[5,6]$. In the present study, we found 2.1and 2.9-fold elevation of plasma CXCL13, a specific chemokine ligand for CXCR5, among primary and SLErelated AIHA patients, respectively. Meanwhile, plasma cytokines hallmarked for established Th subgroups including IFN- $\gamma$, IL-4, and IL-17 were not found elevated in either AIHA genre. Follicular helper T cell (Tfh) has recently been acknowledged as an independent $\mathrm{Th}$ population characterized by surface marker CXCR5. Tfh is crucial for the formation and maintenance of germinal center, and the defect of Tfh self-tolerance may be responsible for autoimmune disorders, especially those marked with high level autoantibody production [8]. We supposed that Th might play an important role in the 
Table 2 Plasma cytokine portraits of primary and SLE-related AIHA patients (pg/ml)

\begin{tabular}{|c|c|c|c|}
\hline & Primary $(n=19)$ & SLE-related $(n=18)$ & Health control $(n=10)$ \\
\hline $\mathrm{IFN}-\gamma$ & $30.93(19.53-45.66)$ & $36.83(26.72-69.23)$ & $48.78(38.10-61.94)$ \\
\hline I-309/CCL1 & $65.26(38.62-108.86)^{*}$ & $123.78(81.56-192.06)$ & $179.66(96.27-194.22)$ \\
\hline MCP-1/CCL2 & $557.52(366.62-709.14) *$ & $683.57(546.29-1070.0) ※$ & $284.96(200.73-372.66)$ \\
\hline MIP-1a/CCL3 & $77.23(64.74-119.83)$ & $114.56(78.54-162.82)$ & $115.37(93.05-133.28)$ \\
\hline MIP-1 $\beta / C C L 4$ & $23.73(12.38-38.30)^{※}$ & $18.75(11.11-33.73)^{※}$ & $5.05(4.02-10.22)$ \\
\hline MIP-18/CCL15 $\left(\times 10^{4}\right)$ & $0.88(0.72-1.16)$ & $0.55(0.29-1.05)$ & $1.37(0.56-1.85)$ \\
\hline RANTES/CCL5 $\left(\times 10^{3}\right)$ & $4.12(2.40-7.38)$ & $5.89(1.52-16.33)$ & $5.03(1.94-7.24)$ \\
\hline Eotaxin/CCL11 & $95.41(64.34-138.11)$ & $118.44(91.65-166.06)$ & $120.81(92.81-133.95)$ \\
\hline Eotaxin-2/CCL24 & $623.34(329.62-810.59)^{\star}$ & $412.51(282.29-720.39)$ & $289.56(206.08-370.20)$ \\
\hline $\mathrm{MIG} / \mathrm{CXCL} 9\left(\times 10^{3}\right)$ & $2.89(1.95-5.15)$ & $3.11(2.02-10.26)$ & $2.36(1.82-4.12)$ \\
\hline BLC/CXCL13 & $147.90(104.11-436.02)^{*}$ & $203.45(110.74-290.42)^{※}$ & $69.14(56.24-100.39)$ \\
\hline ICAM-1 $\left(\times 10^{5}\right)$ & $1.34(0.66-1.99)$ & $0.60(0.49-0.93)^{※}$ & $1.88(0.95-2.73)$ \\
\hline G-CSF & $11.29(4.15-24.12)$ & $16.44(12.83-22.74)$ & $25.12(14.47-28.15)$ \\
\hline M-CSF & $35.48(19.14-66.10) ※$ & $82.47(29.22-181.19)^{\#}$ & $122.47(71.49-150.85)$ \\
\hline GM-CSF & $36.70(23.65-48.62)$ & $41.60(25.59-78.01)$ & $48.22(39.60-72.10)$ \\
\hline IL-1a & $7.51(3.00-26.10)^{\circledast}$ & $27.28(7.85-60.07)^{\#}$ & $42.77(24.27-53.30)$ \\
\hline$I L-1 \beta$ & $63.98(40.83-159.84)$ & $133.24(89.85-223.31)^{\#}$ & $203.08(98.42-223.41)$ \\
\hline IL-1ra & $202.27(121.20-368.22)$ & $416.94(192.30-563.72)$ & $121.82(89.31-196.76)$ \\
\hline $\mid \mathrm{L}-2$ & $9.04(5.14-12.20)$ & $9.81(6.83-18.59)$ & $11.12(9.43-13.68)$ \\
\hline $\mid \mathrm{L}-4$ & $8.98(6.04-16.53)$ & $25.87(8.61-67.04)^{\#}$ & $29.64(13.45-35.24)$ \\
\hline IL-5 & $67.17(46.65-116.94) *$ & $104.56(55.58-155.07)$ & $150.04(103.54-176.69)$ \\
\hline IL-6 & $52.20(40.23-76.39)$ & $71.41(40.80-106.07)$ & $62.45(52.92-72.92)$ \\
\hline IL-6sR $\left(\times 10^{3}\right)$ & $4.05(3.49-4.70)$ & $4.31(3.45-5.17)$ & $4.57(3.78-4.86)$ \\
\hline IL-7 & $72.16(44.68-115.49)$ & $85.55(59.18-147.61)$ & $81.55(72.15-110.65)$ \\
\hline IL-8/CXCL8 & $8.66(5.81-26.17)^{*}$ & $19.15(9.23-26.68) ※$ & $4.84(3.95-7.21)$ \\
\hline $\mid \mathrm{L}-10$ & $26.68(17.98-42.12)$ & $37.50(19.68-71.93)$ & $15.22(14.40-26.26)$ \\
\hline $\mid \mathrm{L}-11$ & $205.97(61.19-342.55)^{※}$ & $324.66(174.49-546.81)^{※}$ & $666.37(487.65-861.28)$ \\
\hline IL-12p40 & $301.15(83.39-558.00)$ & $570.79(234.36-1207.2)$ & $277.58(151.29-403.68)$ \\
\hline IL-12p70 & $4.96(2.52-9.90)^{\star}$ & $16.41(4.91-27.76)^{\#}$ & $17.04(10.93-22.00)$ \\
\hline IL-13 & $6.82(4.40-9.99) *$ & $10.18(5.87-14.10)$ & $10.87(10.53-14.63)$ \\
\hline $\mid \mathrm{L}-15$ & $149.92(61.75-478.57)$ & $130.10(87.61-216.20)$ & $194.64(169.31-233.75)$ \\
\hline IL-16 & 127.04 (87.19-188.99) & $187.65(105.95-365.92)$ & $168.00(102.51-214.64)$ \\
\hline IL-17 & $89.62(36.65-197.82) *$ & $184.42(78.67-298.60)^{*}$ & $438.99(279.09-667.12)$ \\
\hline TIMP-1 $\left(\times 10^{4}\right)$ & $1.15(0.87-1.46)$ & $1.63(0.77-3.29)$ & $2.18(1.13-3.67)$ \\
\hline TIMP-2 $\left(\times 10^{4}\right)$ & $1.62(1.37-1.78)$ & $1.97(1.40-2.56)$ & $1.97(1.66-2.26)$ \\
\hline PDGF-BB $\left(\times 10^{3}\right)$ & $2.45(1.79-2.83)$ & $2.39(1.99-3.00)$ & $1.56(1.44-2.00)$ \\
\hline TNFa & $57.42(31.45-106.25)$ & $85.66(36.50-121.91)$ & 104.09 (87.59-148.24) \\
\hline TNF $\beta$ & $28.24(26.75-71.80)^{※}$ & $46.24(27.84-128.56)$ & $92.78(64.99-144.54)$ \\
\hline STNFRI $\left(\times 10^{3}\right)$ & $3.41(2.63-3.68)^{*}$ & $4.43(2.67-5.18)^{※}$ & $1.21(0.89-1.72)$ \\
\hline sTNFRII $\left(\times 10^{3}\right)$ & $3.30(2.12-4.99)^{*}$ & $6.99(3.84-11.24)$ ※\# & $2.05(1.37-2.70)$ \\
\hline
\end{tabular}

Data are presented as median (interquartile range). The level of significance for pairwise comparisons was adjusted when multiple comparisons were performed $(p=0.05 / 3=0.017)$.

$\circledast_{p}<0.017$ compared to health control; ${ }^{p}<0.017$ compared to primary AIHA. 

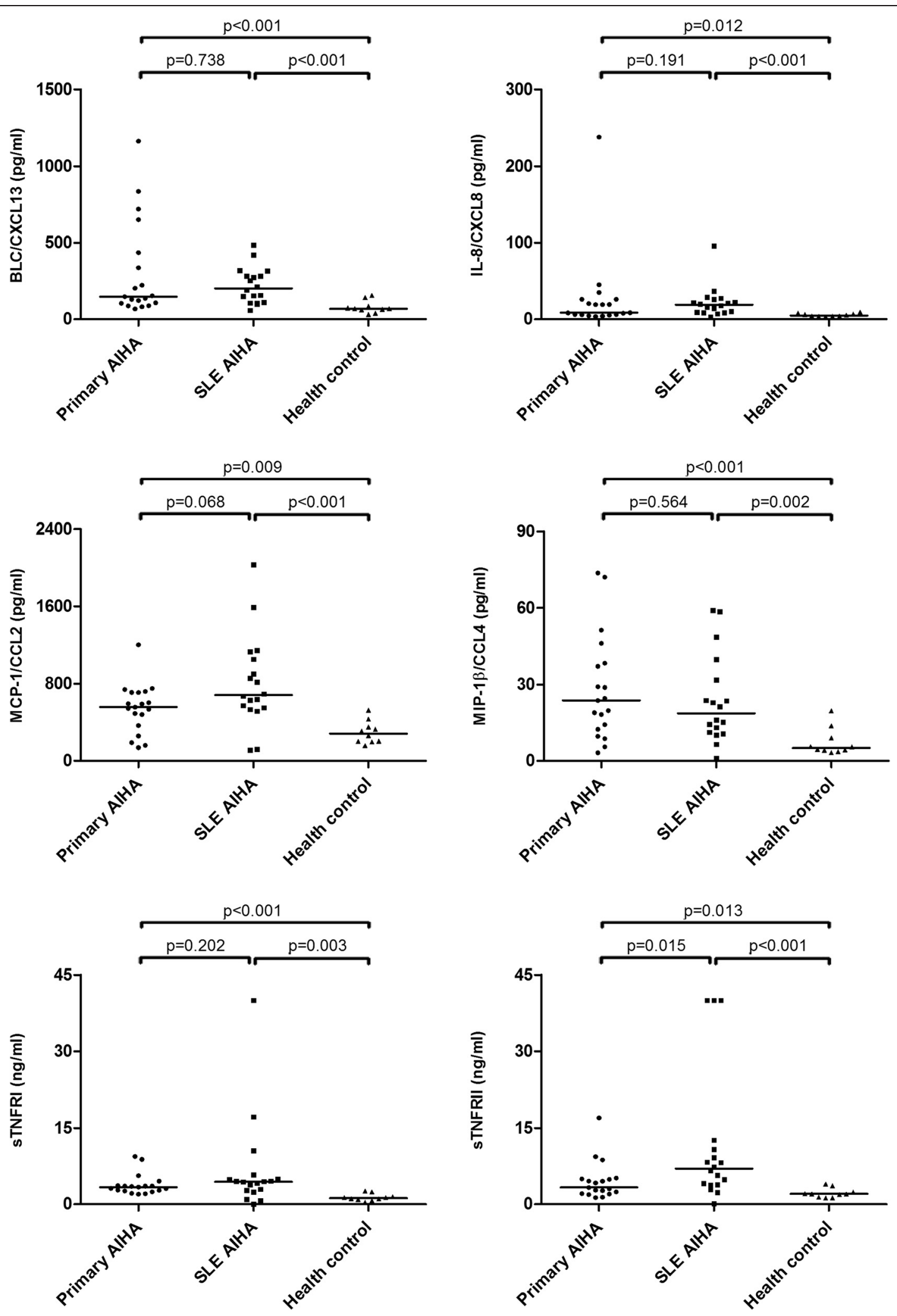

Figure 1 Plasma level of CXCL13, CXCL8, CCL2, CCL4, sTNFRI, and sTNFRII in primary AIHA, SLE-related AIHA, and health control groups. The level of significance for pairwise comparisons was adjusted when multiple comparisons were performed $(p=0.05 / 3=0.017)$. 


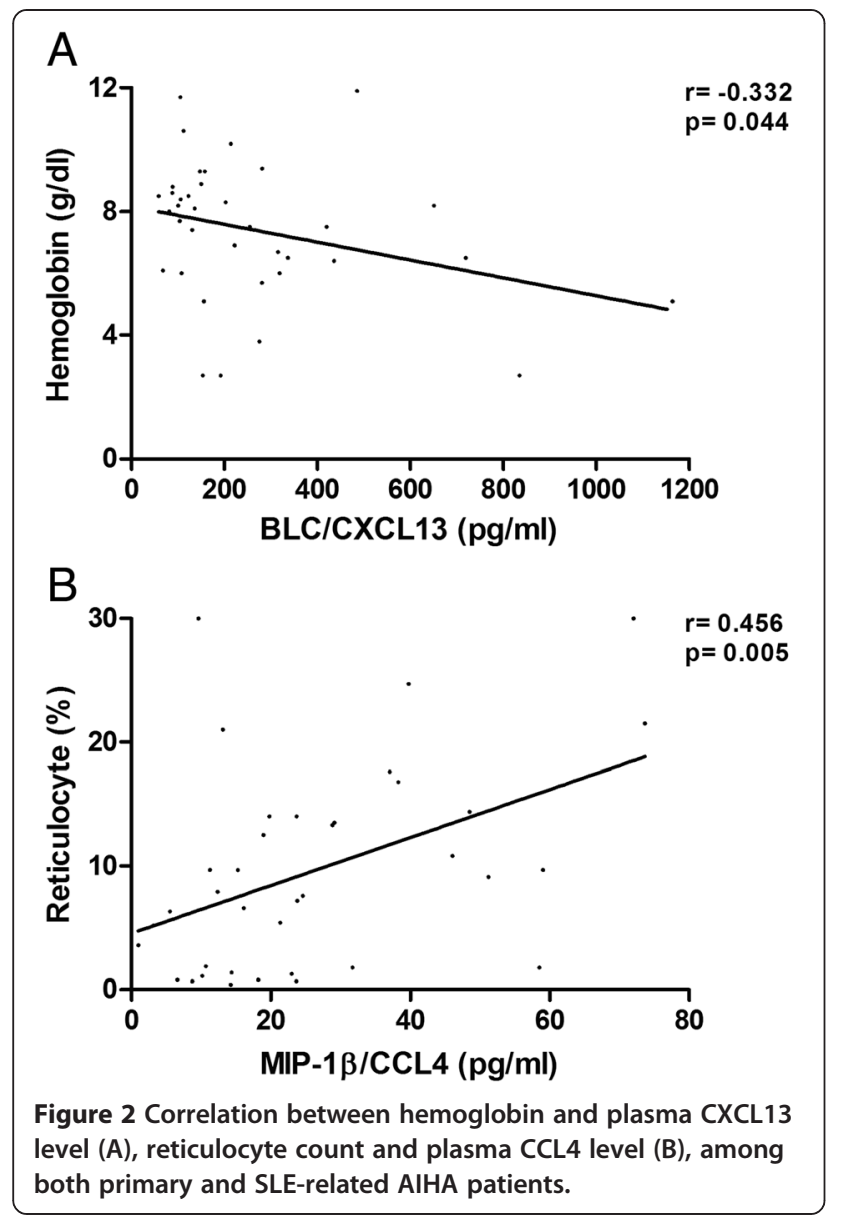

process of autoantibody production among AIHA patients, and CXCL13 could emerge as a potential circulating marker for disease severity.

CCL2, CCL4, CXCL8, and sTNFRI upregulation represented common immune aberration in addition to CXCL13 in both primary and SLE-related AIHA. Interestingly, unlike CXCL13 whose plasma level reflected severe disease, CCL4 might serve as an indicator for robust bone marrow compensation among AIHA patients. Known as macrophage inflammatory protein (MIP)-1 $\beta$, CCL4 closely interacted with MIP- $1 \alpha /$ CCL3 which had been proved to inhibit the proliferation of immature erythroid progenitors $[9,10]$. Therefore, elevated level of circulating CCL4 instead of CCL3 among AIHA patients might indicate a boost in their erythroid proliferation and reticulocytosis. On the other hand, CCL2 and CXCL8, both capable of inducing respiratory burst as well as being chemoattractants, had been under intensive investigation among SLE patients [11-18]. Although both cytokines were argued to be elevated in the scenario of SLE, CCL2 was regarded as an important biomarker for lupus nephritis flair [13-16], and CXCL8 for lupus associated interstitial lung diseases $[17,18]$. In immune-mediated hemolytic anemia canine models,
CCL2 was also argued to experience marked upregulation and to demonstrate prognostic value [19].

The most intriguing finding of the present study was the subtly different patterns of soluble TNFR between primary and SLE-related AIHA patients. Human TNFRII expressed restrictively upon certain $\mathrm{T}$ cell populations and a few other cell types, while TNFRI was found on almost all cell types. The binding of TNF to these two types of receptors generated completely opposite cell fates, with signaling via TNFRI favoring apoptosis and TNFRII survival $[20,21]$. Various defects in the TNFRII pathway, including upregulated expression of TNFRII and TNFRII shedding, had been implicated in SLE patients [22,23]. Based upon the 3.4-fold increase in plasma sTNFRII level, our data supported the hypothesis of TNFRII pathway defect in SLE-related but not primary AIHA. The reason for the elevation of plasma sTNFRI in both AIHA groups was still unclear, since neither TNFR type was expressed on erythrocytes [20].

From the clinical aspect of view, differential diagnosis between primary and secondary AIHA is often challenging, especially among those with positive antinuclear antibody. Aside from younger age and slight female gender preference, SLE-related AIHA seemed to demonstrate less prominent erythrocyte destruction as indicated by serum bilirubin and LDH levels, which could be attributed to impaired scavenging function of reticulo-endothelial system previously described in SLE [24]. Instead of hypocomplementemia which was traditionally believed to be an important diagnostic clue of SLE, the presence of anticardiolipin antibody and upregulation of plasma sTNFRII might be of greater diagnostic value for SLE-related AIHA.

The present study should be viewed in the light of its limitations. It is usually hard to give an exact significance to lab findings concerning clinical situations of high heterogeneity such as SLE. Both disease activity and precedent therapeutic methods could strongly influence lab findings. The study population was relatively small due to low incidence rate of AIHA. The descriptive nature of the present study withheld affirmative causal deduction, and further functional studies could help to clarify the detailed mechanism of specific cytokine induced signaling in AIHA.

\section{Conclusions}

The timely differential diagnosis between primary and SLE-related AIHA often looms as practical challenge under clinical scenario, given the key underlying immune aberration of AIHA remains unclear. The present study performed circulating inflammatory cytokine profiling among primary and SLE-related AIHA patients and revealed novel cytokine patterns in the pathogenesis of AIHA as well as parameters for clinical identification 
of SLE-related patients. Plasma CXCL13 and CCL4 could act as circulating biomarkers in AIHA, and indicated disease severity and erythroid compensation, respectively. Detection of anticardiolipin antibody and higher levels of plasma sTNFRII might favor the diagnosis of SLE-related instead of primary AIHA.

\begin{abstract}
Abbreviations
AlHA: Autoimmune hemolytic anemia; SLE: Systemic lupus erythematosus; Tfh: Follicular helper T cell; CCL: CC chemokine ligand; CXCL: CXC chemokine ligand; IFN: Interferon; MCP: Monocyte chemotactic protein; MIP: Macrophage inflammatory protein; RANTES: Regulated on activation, Normal T cell expressed and secreted; Eotaxin: Eosinophil chemotactic protein; MIG: Monokine induced by gamma interferon; BLC: B lymphocyte chemoattractant; G-CSF: Granulocyte colony stimulating factor; MCSF: Macrophage colony stimulating factor; GM-CSF: Granulocytemacrophage colony stimulating factor; ICAM: Intercellular adhesion molecule; IL: Inerleukin; TIMP: Tissue inhibitor of metalloproteinases; PDGF-BB: Platelet derived growth factor subunit B; TNF: Tumor necrosis factor; sTNFR: Soluble tumor necrosis factor receptor.
\end{abstract}

\section{Competing interests}

The authors declare that they have no competing interests.

\section{Authors' contributions}

Study design: BW, YC; plasma sample collection and multiplex cytokine array analysis: YZ; clinical data collection: WW, SZ, FL; revision and final approval of the manuscript: YC, LS. All authors read and approved the final manuscript.

\section{Acknowledgements}

The study was supported by the National Natural Science Foundation of China $(81170473,81470282)$ and Shanghai Municipal Health Bureau (20144Y0194).

\section{Author details}

'Department of Hematology, Zhongshan Hospital Fudan University, Shanghai 200032, China. ${ }^{2}$ Department of Transfusion, Zhongshan Hospital Fudan University, Shanghai 200032, China. ${ }^{3}$ Biomedical Research Center, Zhongshan Hospital Fudan Universiy, Shanghai 200032, China. ${ }^{4}$ Department of Hematology, Zhongshan Hospital Qingpu Branch, Fudan Universiy, Shanghai 201700, China.

Received: 5 January 2015 Accepted: 23 March 2015

\section{Published online: 08 April 2015}

\section{References}

1. Petz LD, Garratty G. Classification and clinical characteristics of autoimmune hemolytic anemias. In: Petz LD, Garratty G, editors. Immune Hemolytic Anemias. 2nd ed. Philadelphia: Elsevier; 2004. p. 61-73.

2. Packman $\mathrm{CH}$. Hemolytic anemia due to warm autoantibodies. Blood Rev. 2008:22:17-31.

3. Petz LD. Cold antibody autoimmune hemolytic anemias. Blood Rev. 2008;22:1-15.

4. Fagiolo E, Toriani-Terenzi C. Th1 and Th2 cytokine modulation by IL-10/LL-12 imbalance in autoimmune haemolytic anaemia (AlHA). Autoimmunity. 2002:35:39-44.

5. Ward FJ, Hall AM, Cairns LS, Leggat AS, Urbaniak SJ, Vickers MA, et al. Clonal regulatory $T$ cells specific for a red blood cell autoantigen in human autoimmune hemolytic anemia. Blood. 2008;111:680-7.

6. Hoyer KK, Kuswanto WF, Gallo E, Abbas AK. Distinct roles of helper T-cell subsets in a systemic autoimmune disease. Blood. 2009;113:389-95.

7. American College of Rheumatology Ad Hoc Committee on Systemic Lupus Erythematosus Guidelines. Guidelines for referral and management of systemic lupus erythematosus in adults. Arthritis Rheum. 1999;42:1785-96.

8. Tangye SG, Ma CS, Brink R, Deenick EK. The good, the bad and the ugly $T_{F H}$ cells in human health and disease. Nat Rev Immunol. 2013;13:412-26.

9. Su S, Mukaida N, Wang J, Zhang Y, Takami A, Nakao S, et al. Inhibition of immature erythroid progenitor cell proliferation by macrophage inflammatory protein-1 a by interacting mainly with a C-C chemokine receptor, CCR1. Blood. 1997;90:605-11.
10. Kamin-Lewis R, Abdelwahab SF, Trang C, Baker A, DeVico AL, Gallo RC, et al. Perforin-low memory CD8+ cells are the predominant T cells in normal humans that synthesize the $\beta$-chemokine macrophage inflammatory protein-1ß. Proc Natl Acad Sci U S A. 2001;98:9283-8.

11. Lit LC, Wong CK, Tam LS, Li EK, Lam CW. Raised plasma concentration and ex vivo production of inflammatory chemokines in patients with systemic lupus erythematosus. Ann Rheum Dis. 2006;65:209-15.

12. Tuller T, Atar S, Ruppin E, Gurevich M, Achiron A. Common and specific signatures of gene expression and protein-protein interactions in autoimmune diseases. Genes Immun. 2013;14:67-82.

13. Rovin $B H$, Song $H$, Birmingham DJ, Hebert LA, Yu CY, Nagaraja HN. Urine chemokines as biomarkers of human systemic lupus erythematosus activity. J Am Soc Nephrol. 2005;16:467-73.

14. Li Y, Tucci M, Narain S, Barnes EV, Sobel ES, Segal MS, et al. Urinary biomarkers in lupus nephritis. Autoimmun Rev. 2006:5:383-8.

15. El-Shehaby A, Darweesh H, El-Khatib M, Momtaz M, Marzouk S, El-Shaarawy $\mathrm{N}$, et al. Correlations of urinary biomarkers, TNF-like weak inducer of apoptosis (TWEAK), osteoprotegerin (OPG), monocyte chemoattractant protein-1 (MCP-1), and IL-8 with lupus nephritis. J Clin Immunol. 2011;31:848-56.

16. Singh RG, Usha, Rathore SS, Behura SK, Singh NK. Urinary MCP-1 as diagnostic and prognostic marker in patients with lupus nephritis flare. Lupus. 2012;21:1214-8.

17. Al-Mutairi S, Al-Awadhi A, Raghupathy R, Al-Khawari H, Sada P, Al-Herz A, et al. Lupus patients with pulmonary involvement have a pro-inflammatory cytokines profile. Rheumatol Int. 2007;27:621-30.

18. Nielepkowicz-Goździńska A, Fendler W, Robak E, Kulczycka-Siennicka L, Górski P, Pietras T, et al. Exhaled IL-8 in systemic lupus erythematosus with and without pulmonary fibrosis. Arch Immunol Ther Exp (Warsz). 2014;62:231-8

19. Kjelgaard-Hansen $M$, Goggs $R$, Wiinberg B, Chan DL. Use of serum concentrations of interleukin-18 and monocyte chemoattractant protein-1 as prognostic indicators in primary immune-mediated hemolytic anemia in dogs. J Vet Intern Med. 2011;25:76-82.

20. Faustman D, Davis M. TNF receptor 2 pathway: drug target for autoimmune diseases. Nat Rev Drug Discov. 2010;9:482-93.

21. Soloviova K, Puliaiev M, Haas M, Via CS. In-vivo maturation of allo-specific CD8 CTL and prevention of lupus-like graft-versus-host disease is critically dependent on T cell signaling through the TNF p75 receptor but not the TNF p55 receptor. J Immunol. 2013;190:4562-72.

22. Komata T, Tsuchiya N, Matsushita M, Hagiwara K, Tokunaga K. Association of tumor necrosis factor receptor 2 (TNFR2) polymorphism with susceptibility to systemic lupus erythematosus. Tissue Antigens. 1999;53:527-33.

23. Gabay C, Cakir N, Moral F, Roux-Lombard P, Meyer O, Dayer JM, et al. Circulating levels of tumor necrosis factor soluble receptors in systemic lupus erythematosus are significantly higher than in other rheumatic diseases and correlate with disease activity. J Rheumatol. 1997;24:303-8.

24. Frank MM, Hamburger MI, Lawley TJ, Kimberly RP, Plotz PH. Defective reticuloendothelial system Fc-receptor function in systemic lupus erythematosus. N Engl J Med. 1979;300:518-23.

\section{Submit your next manuscript to BioMed Central and take full advantage of:}

- Convenient online submission

- Thorough peer review

- No space constraints or color figure charges

- Immediate publication on acceptance

- Inclusion in PubMed, CAS, Scopus and Google Scholar

- Research which is freely available for redistribution 